\title{
ENFERMEDADES RARAS Y DISCAPACIDAD INTELECTUAL: EVALUACIÓN DE LA CALIDAD DE VIDA DE NIÑOS Y JÓVENES
}

\section{Rare diseases and intellectual disability: assessment of quality of life of children and adolescents}

\author{
Erica GonZÁlez MarTín \\ erika13@hotmail.com \\ Laura E. GÓMEZ SÁNCHEZ \\ Universidad de Oviedo. Departamento de Psicología \\ M. Ángeles Alcedo Rodríguez \\ Universidad de Oviedo. Departamento de Psicología
}

Universidad de Oviedo. Departamento de Psicología. Plaza de Feijoo, s/n. 33003 Oviedo

Recepción: 3 de febrero de 2016

Fecha de aceptación definitiva: 30 de junio de 2016

Resumen: Antecedentes. El principal objetivo de este trabajo ha sido evaluar la calidad de vida en niños y jóvenes con enfermedades raras y discapacidad intelectual, así como conocer la incidencia de determinadas variables (i. e., género, edad, nivel de discapacidad intelectual, tipo de escolarización, tipo de enfermedad y comunidad autónoma) en la variable criterio. Método. Para ello se ha utilizado la Escala KidsLife, elaborada según el modelo de ocho dimensiones de calidad de vida de Schalock y Verdugo. La muestra estuvo formada por 103 participantes con enfermedades raras y discapacidad intelectual, con edades comprendidas entre 3 y 21 años, que recibían apoyos en alguna organización proveedora de servicios educativos, sociales o sanitarios. Resultados. Las puntuaciones más altas se corresponden con la dimensión bienestar físico, mientras que la dimensión inclusión social es la que obtiene puntuaciones más bajas. El nivel de discapacidad intelectual y el de necesidades de apoyo dieron lugar a diferencias significativas en la puntuación total de la escala. El análisis de cada una de las dimensiones muestra diferencias según el género, nivel de discapacidad intelectual y tipo de escolarización. Conclusión. Los resultados abogan por el diseño de prácticas que permitan la mejora de 
resultados personales de este colectivo en lo relativo a su autodeterminación, inclusión y relaciones interpersonales, principalmente.

PALABRAS Clave: enfermedades raras; discapacidad intelectual; calidad de vida; evaluación.

AвsTRAct: Antecedents. The main objective of this study was to evaluate the quality of life in children and young people with rare diseases and intellectual disability, as well as to determine the incidence of certain predictors (i. e., gender, age, level of intellectual disability, type of school, type of illness and autonomous community) in the criterion variable. Method. The KidsLife Scale was applied, a questionnaire based on the eight domain model of quality of life by Schalock and Verdugo. The sample comprised 103 participants with rare diseases and intellectual disability, aged between 3 and 21, who received supports in any organization providing educational, social, or health services. Results. The best scores were found in physical wellbeing, while the lowest were in social inclusion. The level of intellectual disability and support needs resulted in significant differences for the total score of the scale. Analyses by domains showed differences by gender, intellectual disability level, and type of schooling. Conclusions. The results argue for designing practices aimed to improve quality of life-related personal outcomes with regard to self-determination, inclusion, and interpersonal relationships.

KEYWORDs: rare diseases; intellectual disability; quality of life; assessment.

\section{Introducción}

$\mathrm{E}$ N NUESTRO PAís, EL TOTAL DE PERSONAS CON DisCAPACIDAD, según datos de la Encuesta de Discapacidad, Autonomía personal y situaciones de Dependencia (EDAD; Instituto Nacional de Estadística, 2008), se sitúa alrededor del 8.5\% de la población, ascendiendo a 600 millones de personas cuando nos referimos a población mundial. Conforme a dicha encuesta, en España existen 60.400 niños y niñas con limitaciones, con edades comprendidas entre 0 y 5 años, de los que el $60.3 \%$ son niños y el 39.7\% niñas; y 78.300 niños y niñas con algún tipo de discapacidad entre 6 y 15 años, de los que el 64.6\% son niños y el 35.4\% niñas. Para los niños y las niñas con edades comprendidas entre 16 y 18 años no existen datos desglosados (Unicef, 2013a).

Cuando nos referimos al colectivo de personas afectadas por enfermedades raras (ER) las cifras de prevalencia son más bajas, aun así en España se estima que pueden afectar a tres millones de personas (Federación Española de Enfermedades Raras, FEDER, 2015). Como cabe imaginar, hay ER que afectan a un reducido número de personas en el mundo, pero que de manera conjunta aquejan a más de 30 millones de ciudadanos de la Unión Europea (European Union Committee of Experts on Rare Diseases, 2014) y a un número similar en EE. UU. y América Latina. Por ello, diversos autores (López et al., 2012; Puente-Ferreras, Barahona-Gomariz y Fernández-Lozano, 2011) consideran que las ER representan un problema de salud pública. La mayoría de ellas son desconocidas para la población, ya que afectan a menos de 5 de cada 10.000 personas. Además, algunas son frecuentes en una parte del mundo, pero infrecuentes en otros lugares. Sin embargo, la Organización Mundial de la Salud

(C) Ediciones Universidad de Salamanca / CC BY-NC-ND

Siglo Cero, vol. 47 (3), n. ${ }^{\circ} 259,2016$, julio-septiembre, pp. 7-27 
(OMS) reconoce más de 7.000 enfermedades denominadas raras, que afectan al 7\% de la población mundial en sus capacidades físicas, habilidades mentales y en sus destrezas sensoriales y de comportamiento (FEDER, 2015).

En términos generales, se entiende que las ER son en su mayoría enfermedades crónicas que producen una elevada mortalidad y morbilidad prematura, además de un alto grado de discapacidad y, por lo tanto, un gran deterioro de la calidad de vida (CV) de los afectados debido al aislamiento, invisibilidad e impotencia (Caputo, 2014; López et al., 2012; Sprangers et al., 2000). En este sentido, la red epidemiológica de investigación en enfermedades raras incorporó a la definición la presencia de al menos una de las siguientes características: escaso conocimiento etiológico, cronicidad, falta de tratamiento curativo o de baja accesibilidad, importante carga de la enfermedad o limitación de la calidad de vida (Puente-Ferreras et al., 2011).

Desde esta perspectiva es importante señalar las diferencias existentes entre conceptos que, en ocasiones, pueden conducir a error si no se utilizan de forma adecuada. No son pocas las ocasiones en que el término "enfermedad olvidada" se utiliza como sinónimo de ER, cuando en realidad una enfermedad olvidada es aquella que está desdeñada por la industria farmacéutica ya que no es una prioridad de salud pública (p. e., enfermedades infecciosas frecuentes en países subdesarrollados, pero poco frecuentes en nuestro medio). Las enfermedades olvidadas no son, por tanto, ER. Del mismo modo que las "enfermedades huérfanas" incluyen tanto a las ER y a las enfermedades olvidadas. Se denominan así por estar huérfanas de atención por parte de la investigación y de interés del mercado y políticas de salud pública (Puente-Ferreras et al., 2011). Por último, cabe destacar la diferencia que existe entre ER y enfermedades crónicas. Aunque ambas pueden ser de origen genético, tener un curso crónico y degenerativo, con consecuencias negativas a nivel socioeconómico, la diferencia radica en el conocimiento médico y la identidad, que en el caso de las ER suele ser más tardío y conllevar una mayor dificultad diagnóstica en comparación con las enfermedades crónicas (Picci et al., 2015). En muchos casos no se conoce exactamente cuál es la causa de la enfermedad. En otros puede deberse a mutaciones de un único gen $(80 \%$ de los casos), factores medioambientales (dieta, tabaco, exposición a agentes químicos) o agentes infecciosos. En la mayoría de los casos los síntomas de las ER aparecen al nacer o durante la infancia, aunque existen ocasiones en las que los síntomas se hacen visibles en la etapa adulta.

Como se ha mencionado anteriormente, la problemática asociada a las ER es variada. En primer lugar, uno de los problemas de las familias es obtener un diagnóstico, que en ocasiones puede ser precoz si los síntomas son evidentes, pero que por norma general suele ser tardío debido a la falta de especificidad clínica, su comienzo insidioso y la afectación multisistémica que desorienta al clínico.

En segundo lugar, otro problema al que se enfrentan las familias de niños con ER es la falta de información sobre la enfermedad, así como la insuficiente coordinación entre los diferentes profesionales y niveles asistenciales implicados en la atención de sus hijos (Gaite, García Fuentes, González Lamuño y Álvarez, 2008).

En tercer lugar, la disponibilidad de tratamiento fue uno de los elementos que generó el movimiento asociativo relacionado con las ER ya que, en términos generales, 
las opciones terapéuticas son escasas y poco eficaces. Todo ello conduce a los denominados "medicamentos huérfanos", que son aquellos medicamentos que, aun siendo de elevado interés terapéutico, no están disponibles debido a que no se comercializan o no están indicados para el problema específico de salud. Según Puente-Ferreras et al. (2011), este aspecto se relaciona directamente con la industria farmacéutica, ya que antes de invertir en $\mathrm{I}+\mathrm{D}$ para un producto debe calcular si se recuperará la inversión con las ventas.

Por último, dado que las ER no afectan sólo a la persona que padece la enfermedad, sino también a sus familiares, se producen numerosas dificultades asociadas, como puede ser la carga económica a afrontar que puede llegar a ser elevada, así como la dificultad de al menos uno de los padres para seguir manteniendo su empleo debido a la demanda de atención continuada. De este modo, los padres de niños con ER suelen ver reducida su CV y bienestar, lo que a su vez también puede repercutir en el cuidado de sus hijos (Johansen, Dammann, Andresen y Fagerland, 2013).

En términos generales, a pesar de que las ER constituyen un grupo muy heterogéneo, colocan a los pacientes y sus familias ante un amplio abanico de necesidades: incertidumbre en cuanto al diagnóstico, disponibilidad de tratamientos efectivos, administración de las medicaciones prescritas, alteraciones en su conducta social y laboral, impacto emocional de los síntomas a afrontar, discapacidad y riesgo de muerte anticipada (Gaite et al., 2008). Tal es así, que durante las dos últimas décadas los investigadores han destacado la necesidad de evaluar la $\mathrm{CV}$ de los niños y niñas con ER, con el objetivo de determinar el impacto que estas enfermedades y los tratamientos tienen sobre sus vidas (Caliandro et al., 2007). Vivir con enfermedades y discapacidad puede afectar a la vida diaria en muchos aspectos. Algunas personas con discapacidad requieren atención médica continuada y necesitan adaptaciones individuales y específicas en múltiples áreas. Además, en el caso de los niños, implica una reducción de las relaciones, debido a las dificultades que pueden tener para relacionarse con sus iguales, así como una disminución de las actividades en las que pueden participar, lo que merma significativamente su CV (Johansen et al., 2013). Cuando a la presencia de una ER se añade la comorbilidad con discapacidad intelectual (DI), la independencia funcional se encuentra limitada y las habilidades para la vida diaria se ven disminuidas en comparación con sus pares sin discapacidad (Bendixen, Senesac, Lott y Vanderborne, 2012).

Todo lo anterior pone de relieve la importancia de la evaluación e intervención en lo relativo a la $\mathrm{CV}$ en las personas con discapacidades múltiples, severas y profundas, cuyo estudio aún constituye un reto por abordar (Gómez et al., 2014). No obstante, gracias a los avances en la operacionalización del constructo en los colectivos con mayores niveles de funcionamiento, podemos operativizar el constructo en las personas que necesitan mayor intensidad y calidad de apoyos. De este modo, la evaluación de la CV se está convirtiendo en una forma interesante y novedosa de obtener información acerca de la experiencia de la enfermedad, la eficacia de los tratamientos y de la necesidad de cuidados y apoyos intensos, generalizados y complejos (Gómez et al., 2016a; Mura, Krishna, Pisano, Licci y Carta, 2012).

El término CV es un concepto evolucionado en los últimos años desde una noción sensibilizadora hasta convertirse en un agente de cambio que guía las prácticas de

(C) Ediciones Universidad de Salamanca / CC BY-NC-ND

Siglo Cero, vol. 47 (3), n. ${ }^{\circ} 259,2016$, julio-septiembre, pp. 7-27 
profesionales y servicios preocupados por las personas en riesgo de exclusión social (Gómez, Verdugo, Arias y Navas, 2010a). Antes de los años 60, sólo existían conceptos relacionados con la $\mathrm{CV}$ que hoy consideramos lejanos por basarse únicamente en medidas objetivas y normativas (p. e., "renta per cápita", "nivel de vida”). Desde ese momento, el concepto fue experimentando una evolución que implicó, a su vez, introducir la perspectiva de satisfacción con la vida, bienestar y los sentimientos de felicidad, de forma que se orientaba más hacia una perspectiva centrada en la persona. Después, durante los años 80 , se produjeron numerosos avances en este campo, en gran medida por el auge de los movimientos en defensa de los derechos humanos y el creciente interés reflejado en la legislación por promover la calidad. Posteriormente, ya en la década de los 90, el trabajo se centró principalmente en la especificación, medición e integración del constructo de $\mathrm{CV}$ en las prácticas profesionales.

Esencialmente, es el carácter dinámico del constructo el que hace que su conceptualización sea un reto difícil, ya que no existe una definición unánime de $\mathrm{CV}$, sino más de un centenar de definiciones y de un millar de instrumentos de evaluación (Alcedo, Aguado, Arias, González y Rozada, 2008; Gómez, Verdugo y Arias, 2010b). Actualmente, y dado que el objetivo por conseguir una definición ampliamente consensuada que sea aceptada por todos sigue siendo un reto aún no logrado, la comunidad científica ha optado por la creación de un marco conceptual que unifique las dimensiones y los indicadores de una vida de calidad. De esta forma, surgen los modelos operativos, cuyo objetivo es facilitar la comprensión de la CV mediante la identificación de factores centrales o dimensiones de CV (Gómez, 2010).

En este sentido, son varios los modelos que han tratado de operativizar el constructo de CV. En primer lugar, el modelo comprehensivo de CV de Cummins, que aporta una definición operativa para evaluar este constructo desde una perspectiva objetiva y subjetiva a través de siete dimensiones relevantes: (a) bienestar material; (b) salud; (c) productividad; (d) intimidad; (e) seguridad; (f) presencia en la comunidad; y (g) bienestar emocional. Estas dimensiones se consideran aplicables a todas las personas, tengan o no discapacidad. Cummins (2000) propone que estas variables objetivas y subjetivas sean ponderadas en función de la importancia o valor que la persona otorga a cada dimensión, de tal modo que, aunque los factores contextuales ejerzan presión sobre el componente afectivo y cognitivo de la persona, la valoración subjetiva se mantendrá estable gracias a un proceso de homeostasis, que sólo se modificará si existe un acontecimiento contextual suficientemente aversivo.

En segundo lugar, el modelo propuesto por Felce y Perry (1995) trata de integrar la descripción objetiva de las condiciones de vida y la evaluación subjetiva de la satisfacción con la vida, con una tercera dimensión: las aspiraciones personales ("importancia”). Para ello, proponen un modelo con cinco dimensiones (a) bienestar físico; (b) bienestar material; (c) bienestar social; (d) desarrollo y actividad; y (e) bienestar emocional. La relación entre las tres variables y las influencias externas (economía, variables políticas y sociales) son las que determinan la $\mathrm{CV}$ de una persona.

En tercer lugar, el modelo de Petry, Maes y Vlaskamp (2005) consiste en una adaptación del anterior modelo de Felce y Perry a personas con DI profunda y múltiple. Los resultados de su investigación pusieron de manifiesto que los indicadores de las

(C) Ediciones Universidad de Salamanca / CC BY-NC-ND

Siglo Cero, vol. 47 (3), n. ${ }^{\circ}$ 259, 2016, julio-septiembre, pp. 7-27 
dimensiones básicas diferían de las de otros colectivos, ya que emergían indicadores que no aparecían en el modelo original y que, sin embargo, eran importantes para las personas con discapacidades múltiples y profundas debido a su limitación y dependencia en esas áreas. Además, aunque el contenido de las dimensiones de CV era el mismo para las personas con discapacidades leves que para aquellas con discapacidades profundas y múltiples, estas últimas no podían alcanzarlo sin otros apoyos.

Por último, el modelo propuesto por Schalock y Verdugo $(2002 / 2003$, 2007) define la CV como un estado de bienestar personal que: (a) es multidimensional; (b) tiene propiedades universales y propiedades ligadas a la cultura; (c) tiene componentes objetivos y subjetivos; y (d) está influenciado por características personales y factores ambientales. En este sentido, los autores defienden que este constructo hace referencia al grado en que las personas tienen experiencias vitales que valoran; refleja las dimensiones que contribuyen a una vida interconectada; tiene en cuenta el contexto físico, social y cultural que es importante para las personas, e incluye experiencias humanas comunes y experiencias vitales únicas (Schalock, Verdugo y Gómez, 2011).

Ante esta pluralidad de modelos, y dado que la evaluación de la CV requiere estar sustentada en un marco teórico que defina bien el constructo a medir y las conductas observables ligadas al mismo mediante definiciones operativas (Gómez et al., 2010a), en este trabajo adoptamos el modelo de CV de ocho dimensiones de Schalock y Verdugo (2002/2003). Este modelo, además de ser el más utilizado en el contexto español por los proveedores de servicios sociales y educativos, es uno de los más citados en la literatura especializada y de los que cuentan con mayores evidencias empíricas de validez (Gómez, Verdugo, Arias y Arias, 2011; Wang, Schalock, Verdugo y Jenaro, 2010). Asimismo, refleja los objetivos de integración en los programas de atención; ofrece una visión de las personas con discapacidad en términos de autodeterminación, capacitación e igualdad; hace hincapié en los productos y los resultados, y coincide con las expectativas de los receptores de los servicios, quienes deseaban que influyeran significativa y positivamente en su bienestar. De este modo, el modelo facilita la mejora del bienestar de las personas en sus contextos culturales y reúne los criterios que un modelo teórico útil debe cumplir, proporciona una perspectiva amplia frente a otros que se centran en aspectos concretos de la persona y proporciona indicaciones sobre su utilidad y aplicación para evaluar y mejorar los resultados personales relacionados con la CV (Gómez et al., 2010b).

El modelo se operativiza en torno a ocho dimensiones, sus correspondientes indicadores centrales y resultados personales. Las dimensiones de CV propuestas son: (a) bienestar emocional, (b) relaciones interpersonales, (c) bienestar material, (d) desarrollo personal, (e) bienestar físico, (f) autodeterminación, (g) inclusión social y (h) derechos. Estas dimensiones se entienden como factores que determinan el bienestar personal y se concretan a través de una serie de indicadores centrales, es decir, percepciones, conductas o condiciones específicas de las dimensiones que reflejan el bienestar de una persona (Schalock y Verdugo, 2002/2003: 34). Del mismo modo, los resultados personales reflejan la evaluación de la situación personal o de las aspiraciones de la persona. Asimismo, estos resultados personales son medidas importantes

(C) Ediciones Universidad de Salamanca / CC BY-NC-ND

Siglo Cero, vol. 47 (3), n. ${ }^{\circ} 259,2016$, julio-septiembre, pp. 7-27 
en áreas como la educación, cuidado de la salud y servicios sociales que sirven no sólo para mejorar el bienestar de la persona, sino también para determinar la efectividad de los programas de intervención (Verdugo, Gómez, Arias, Navas y Schalock, 2014).

En lo que se refiere a la estructura del modelo, se han encontrado dificultades en la validación de su estructura factorial y aún se considera una cuestión que debe seguir siendo investigada. Wang et al. (2010) señalan la posibilidad de que el concepto presente una estructura jerárquica, donde la $\mathrm{CV}$ es un constructo formado por ocho dimensiones de primer orden y uno de segundo orden, mientras Gómez et al. (2011), tras realizar una comparación del ajuste de diferentes soluciones jerárquicas con el modelo inicial, concluyen que es el modelo de ocho dimensiones de primer orden el que ofrece un mejor ajuste en comparación con soluciones más complejas de segundo orden. Del mismo modo, la importancia relativa de las dimensiones varía de unas personas a otras, e incluso a lo largo de la vida de una persona, aunque existe cierto consenso internacional sobre su importancia y estructura (Gómez et al., 2010a; Jenaro et al., 2005; Schalock et al., 2005).

Por último, cabe señalar que aunque el modelo ha tenido grandes implicaciones en el ámbito de la DI, progresivamente se está extendiendo a personas con otro tipo de discapacidades y personas en riesgo de exclusión social y con necesidades de apoyo, como, por ejemplo, personas mayores, personas con problemas de salud mental, personas con drogodependencias, VIH o SIDA (Gómez et al., 2010a).

Así, el objetivo general de este trabajo consiste en analizar la CV de niños y jóvenes con ER y DI, con el fin de aproximarnos a una realidad que anteriormente hemos calificado como huérfana de atención por parte de la investigación y carente de interés para el mercado y las políticas de salud pública. Asimismo, como señalan Gómez et al. (2013), la investigación sobre CV y discapacidad durante la infancia y adolescencia apenas ha tenido desarrollo, ya que los pocos estudios existentes sobre CV en este colectivo se realizan con población adulta y las escalas existentes para evaluar $\mathrm{CV}$ en infancia y adolescencia (CCVA y CVI-CVIP) se dirigen a personas con altos niveles de funcionamiento (Gómez-Vela y Verdugo, 2009; Sabeh, Verdugo, Prieto y Contini, 2009). También interesa conocer la incidencia de determinadas variables predictoras (género, edad, nivel de DI, tipo de escolarización, tipo de enfermedad y comunidad autónoma) en la variable criterio (Gómez, Peña, Arias y Verdugo, 2016).

\section{Método}

\section{Participantes}

En este estudio se contó con la participación de 103 niños y jóvenes con DI y ER asociadas, que recibían atención en un total de 47 centros, tanto públicos como privados, proveedores de servicios a personas con discapacidad. Los criterios para poder formar parte de la muestra eran: (a) tener discapacidad intelectual; (b) tener una edad igual o inferior a 21 años; y (c) estar recibiendo servicios o apoyos en alguna organización de tipo social o educativo. 
En cuanto al género, más de la mitad eran hombres $54.4 \%(n=56)$ y el $45.6 \%$ restante mujeres $(n=47)$. El rango de edad oscilaba entre 3 y 21 años $(M=12.97 ; D T=$ 5.09). Respecto a su distribución en función del nivel de DI, el 6.8\% $(n=7)$ tenía una discapacidad leve, el $29.1 \%(n=30)$ moderada, el $46.6 \%(n=48)$ severa y un $17.5 \%$ $(n=18)$ profunda. Todas las personas evaluadas presentaban además otras condiciones asociadas, consideradas enfermedades raras, tal y como se muestra en la Tabla 1 , siendo la más prevalente el síndrome de X-Frágil (12.6\%).

\begin{tabular}{|l|c|c|}
\hline \multicolumn{3}{|c|}{ TABLA 1. Porcentajes y frecuencia de la ER en la muestra } \\
\hline \multicolumn{1}{|c|}{ Enfad } & Frecuencia & Porcentaje \\
\hline X-Frágil & 13 & 12.6 \\
\hline Otros síndromes raros & 12 & 11.7 \\
\hline Otras cromosomopatías & 12 & 11.7 \\
\hline Síndrome de West & 9 & 11.7 \\
\hline Síndrome de Rett & 7 & 8.7 \\
\hline Síndrome de Angelman & 5 & 6.8 \\
\hline Síndrome de Williams & 4 & 4.9 \\
\hline Esclerosis tuberculosa & 3 & 3.9 \\
\hline Síndrome Cri Du Chat & 3 & 2.9 \\
\hline Síndrome Dandy Walker & 3 & 2.9 \\
\hline Síndrome Idic15 & 3 & 2.9 \\
\hline Síndrome de Lennox-Gastaut & 3 & 2.9 \\
\hline Síndrome de Opitz & 3 & 2.9 \\
\hline Síndrome de Turner & 2 & 1.9 \\
\hline Agenesia del cuerpo calloso & 2 & 1.9 \\
\hline Síndrome de Arnold Chiari & 2 & 1.9 \\
\hline Síndrome de Dravet & 2 & 1.9 \\
\hline Síndrome de Steinert & 1 & 1 \\
\hline Síndrome de Prader-Willi & 1 & 1 \\
\hline Síndrome de Klinefelter & 1 & 1 \\
\hline Síndrome de George & & \\
\hline
\end{tabular}

En lo relativo a la procedencia geográfica de los participantes, casi una tercera parte de la muestra procedía de Andalucía (29.1\%). Un porcentaje considerable de personas procedía de Galicia (11.7\%), Principado de Asturias (11.7\%), Canarias (9.7\%), Comunidad Valenciana (8.7\%), Extremadura (7.8\%), Cantabria (6.8\%) y Castilla la Mancha (4.9\%). Aragón, Cataluña, Comunidad de Madrid y País Vasco estuvieron representadas con el 1.9\% (cada región), mientras el 2\% restante se distribuyó entre Castilla y León y La Rioja.

Las evaluaciones de CV de estos participantes fueron realizadas por 57 respondientes que los conocían suficientemente, pues o bien eran profesionales o familiares.

(C) Ediciones Universidad de Salamanca / CC BY-NC-ND

Siglo Cero, vol. 47 (3), n. ${ }^{\circ} 259,2016$, julio-septiembre, pp. 7-27

$$
-14-
$$


Los requisitos para poder completar la escala consistían en: (a) conocer a la persona evaluada desde hacía al menos seis meses y (b) tener oportunidades de observar a la persona evaluada durante periodos prolongados de tiempo en diversos contextos. En este trabajo, el $51.5 \%$ de los respondientes eran profesores, el 19.4\% madres, el $11.7 \%$ psicólogos/as, el $4.9 \%$ padres, el $3.9 \%$ cuidadores, el $1.9 \%$ trabajadores sociales y el $3 \%$ restante se distribuye entre directores de centros, orientadores escolares y logopedas. Estos evaluadores manifestaron conocer a la persona evaluada en un tiempo que fluctuaba entre 6 meses y 20 años $(M=6.38 ; D T=5.75)$. El rango de edad de los respondientes oscilaba entre 24 y 61 años $(M=43.24 ; D T=7.25)$.

En lo que concierne a los 47 centros en los que estaban escolarizados los participantes, el $75.7 \%$ pertenecían al ámbito urbano y el $24.3 \%$ restante al ámbito rural. En cuanto al tipo de organización, el $72.8 \%$ era concertada, el $20.4 \%$ privada y el $6.8 \%$ pública. En función del servicio que prestan estos centros, el $84.5 \%$ correspondían a servicios educativos, el $14.6 \%$ servicios sociales y el $1 \%$ servicios sanitarios.

\section{Instrumento}

El instrumento utilizado ha sido la escala KidsLife, que evalúa resultados personales relacionados con la CV de menores de 21 años con DI, siempre y cuando se encuentren en el sistema educativo. Esta escala se ha diseñado para la evaluación de personas con discapacidades significativas, pero también puede aplicarse a personas con niveles altos de funcionamiento que cumplan dos requisitos fundamentales: (a) tener DI y (b) una edad inferior a 21 años (Gómez et al., 2016b).

El instrumento consta de un total de 156 ítems organizados en torno a ocho dimensiones: inclusión social (IS), autodeterminación (AU), bienestar emocional (BE), bienestar físico (BF), bienestar material (BM), derechos (D), desarrollo personal (DP) y relaciones interpersonales (RI). Los ítems están enunciados en tercera persona y el formato de respuesta es una escala de frecuencia de cuatro puntos (nunca, a veces, frecuentemente, siempre). Su cumplimentación oscila entre 20 y 40 minutos.

La escala también contiene una presentación que especifica sus características, la población diana y los posibles respondientes, una declaración de consentimiento informado en el que los tutores legales autorizan la participación del menor en el estudio para el desarrollo de la escala y se comprometen a colaborar, datos del informador principal (sexo, fecha de nacimiento, relación con la persona evaluada, tiempo que hace que conoce a la persona y frecuencia de contacto con la misma), datos de la organización (nombre completo, acrónimo, e-mail, localidad, provincia, comunidad autónoma, tamaño del centro, tipo de organización, ámbito y tipo de servicio), datos de la persona evaluada (sexo, fecha de nacimiento, nivel de discapacidad y de dependencia, porcentaje de discapacidad, año de expedición, otras condiciones de la persona evaluada, tipo de escolarización, provincia de residencia y comunidad autónoma) y un apartado final para añadir cualquier información o sugerencia que pueda ser relevante para la evaluación.

Las evidencias de fiabilidad obtenidas en la validación de la escala, con una muestra de 1.074 personas, en la que han participado más de un centenar de organizaciones

(C) Ediciones Universidad de Salamanca / CC BY-NC-ND

Siglo Cero, vol. 47 (3), n. ${ }^{\circ} 259,2016$, julio-septiembre, pp. 7-27 
españolas proveedoras de servicios y apoyos a personas con discapacidad, avalan la consistencia interna de la escala KidsLife, con un alpha de Cronbach = .97 (Gómez, Alcedo, Monsalve y Fontanil, 2014; Gómez et al., 2014; Gómez et al., 2016a, b). En la Figura 1 se muestran los resultados obtenidos en el análisis de fiabilidad de la escala y de las distintas dimensiones a partir de la muestra de este estudio.

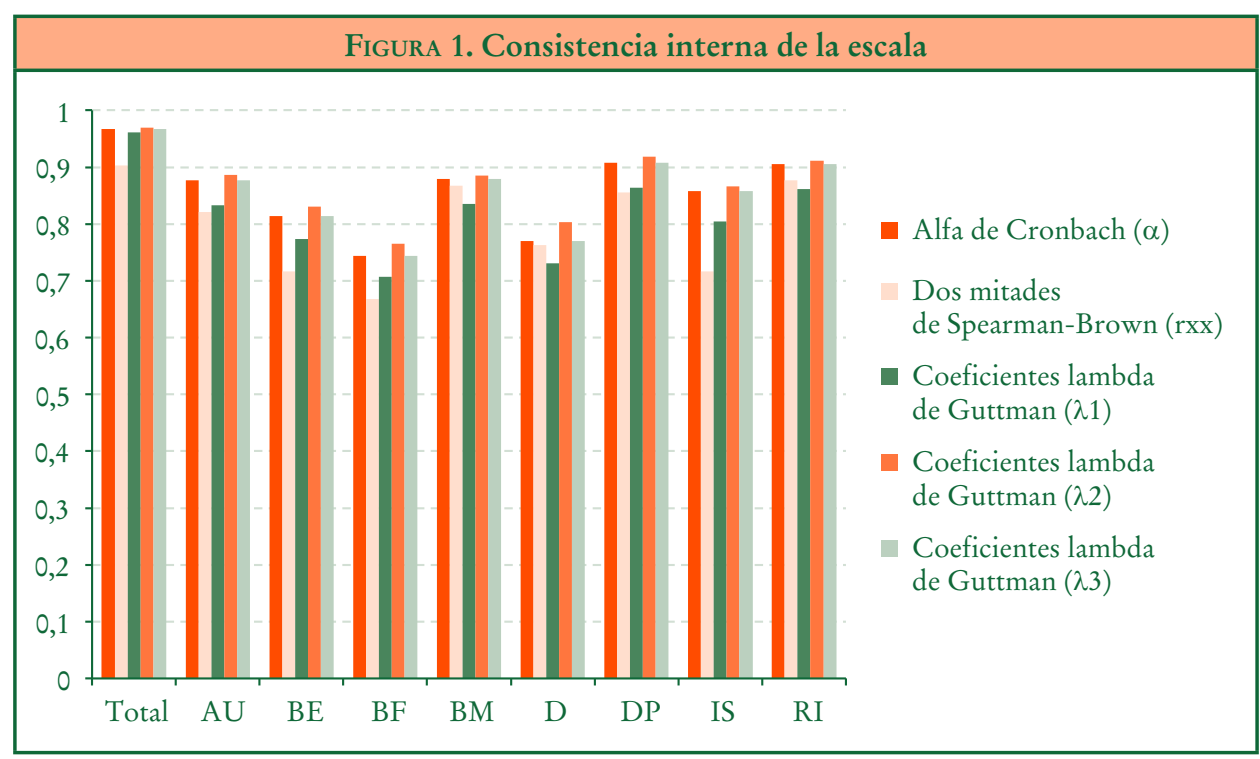

Nota. $\mathrm{AU}=$ autodeterminación; $\mathrm{BE}=$ bienestar emocional; $\mathrm{BF}=$ bienestar físico; $\mathrm{BM}=$ bienestar material; $\mathrm{D}=$ derechos; $\mathrm{DP}=$ desarrollo personal; $\mathrm{IS}$ = inclusión social; $\mathrm{RI}$ = relaciones interpersonales.

\section{Procedimiento}

El reclutamiento de los participantes se llevó a cabo en diferentes ámbitos geográficos siguiendo un riguroso protocolo de administración. En primer lugar, el Grupo de Investigación sobre Discapacidad (GID) (PSI2012-33139) de la Universidad de Oviedo, con el apoyo del Instituto Universitario de Integración en la Comunidad (INICO) de la Universidad de Salamanca, se puso en contacto con diferentes asociaciones y organizaciones mediante una carta o correo electrónico, explicando los objetivos del estudio y solicitando la participación de los mismos.

A continuación, los centros confirmaban durante un plazo establecido si querían participar en el estudio. Si aceptaban colaborar inscribían los datos de la organización en una base informatizada para su posterior uso. Una vez confirmada la participación se envió a cada centro un enlace de acceso a la versión electrónica de la escala y al manual con las instrucciones para cumplimentarla. También se aportaba descripción del proyecto y los objetivos de la investigación, una explicación del concepto de calidad de 
vida y de la necesidad de evaluación de la misma, el proceso de desarrollo de la escala así como las instrucciones necesarias para su cumplimentación y, por último, una dirección de correo y un número de teléfono para que pudieran realizar las consultas oportunas. Cabe destacar que no sólo se solicitó la colaboración vía correo electrónico, sino que también hubo contacto directo con las organizaciones, siendo una estrategia fundamental para que muchos se decidieran a participar.

De este modo, la escala se entregaba al profesional o familiar que iba a cumplimentarla. Primero debían declarar que existía consentimiento informado, responder una serie de datos sociodemográficos tanto de la persona evaluada como del informador principal, para posteriormente responder los 156 ítems que componen la escala KidsLife. La evaluación se llevó a cabo garantizando la confidencialidad de los datos y siguiendo los estándares éticos en la investigación, siendo aprobada por el Comité de Ética de la Universidad de Oviedo.

\section{Análisis de datos}

Para contrastar la relación entre las variables de dos niveles (i. e., género) para la puntuación total, las dimensiones y los ítems, se utilizó la prueba $t$ para muestras independientes, mientras que para aquellas variables con más de dos niveles (i. e., edad, tipo de escolarización, nivel de DI, comunidad autónoma de residencia y nivel de necesidades de apoyo) se realizaron análisis de varianza (ANOVA) y las correspondientes pruebas post hoc (Scheffé y T3 de Dunett). En ambos casos se utilizó un nivel de significación del 95\% $(p<.05)$ y se hallaron los tamaños de los efectos. Se utilizó el paquete estadístico SPSS 15.0 para Windows.

\section{Resultados}

La distribución de las puntuaciones totales obtenidas en la escala KidsLife (Tabla 2) muestra asimetría negativa (asimetría $=-.62$; curtosis $=.16$ ). Las puntuaciones oscilan entre 318 y 579, con una media de $494.72(D T=52.24)$ y mediana de 504. Dado que el rango de respuesta de la escala va de 156 a 654, tanto la media como la mediana superan el punto medio teórico de la misma (Punto Medio Teórico $=312$ ), ya por el percentil 25. La puntuación alcanzada con más frecuencia por los participantes es 504.

\begin{tabular}{|l|c|l|c|}
\hline \multicolumn{4}{|c|}{ TABLA 2. Estadísticos descriptivos de las puntuaciones obtenidas en la escala KidsLife } \\
\hline \multicolumn{4}{|c|}{ Descriptivos Escala KidsLife } \\
\hline Media & 494.72 & Punto medio teórico & 312 \\
\hline E. T. Media & 5.14 & Varianza & 2728.96 \\
\hline N. Válido & 103 & Desviación típica & 52.24 \\
\hline Mediana & 504 & Rango & 261 \\
\hline
\end{tabular}

(C) Ediciones Universidad de Salamanca / CC BY-NC-ND

Siglo Cero, vol. 47 (3), n. ${ }^{\circ}$ 259, 2016, julio-septiembre, pp. 7-27

$$
-17-
$$




\begin{tabular}{|c|c|c|c|}
\hline \multicolumn{4}{|c|}{ Descriptivos Escala KidsLife } \\
\hline Moda & 504 & Percentiles & $\begin{array}{l}459 \\
504 \\
536\end{array}$ \\
\hline Mínimo & 318 & Asimetría & -.62 \\
\hline Máximo & 579 & Curtosis & .16 \\
\hline
\end{tabular}

En cuanto a la distribución de cada una de las dimensiones que conforman la escala KidsLife, cabe precisar que todas ellas están compuestas por 20 ítems cuya puntuación va de 1 a 4, oscilando el rango de respuesta entre 20 y 80, a excepción de la dimensión IS que cuenta con 16 ítems, cuyo rango de respuesta fluctúa entre 16 y 64 . En la Tabla 3 se puede observar que la dimensión que obtiene una media más alta es la de $\mathrm{BF}(M=68.72)$, mientras que la más baja se encontró en IS $(M=41.57)$. Además, la media de cada dimensión supera en todos los casos el punto medio teórico de la subescala.

\begin{tabular}{|l|c|c|c|c|c|c|c|c|}
\hline \multicolumn{8}{|c|}{ TabLA 3. Estadísticos descriptivos de las ocho dimensiones de la escala KidsLife } \\
\hline & IS & AU & BE & BF & BM & D & DP & RI \\
\hline & 16 & 20 & 20 & 20 & 20 & 20 & 20 & 20 \\
\hline N. o ítems & 41.57 & 53.90 & 65.33 & 68.72 & 68.44 & 68.21 & 65.17 & 63.38 \\
\hline Media & 8.00 & 9.60 & 7.88 & 6.66 & 9.03 & 6.39 & 9.30 & 10.36 \\
\hline DT & 19 & 26 & 45 & 53 & 28 & 55 & 37 & 28 \\
\hline Mínimo & 61 & 76 & 80 & 80 & 80 & 80 & 80 & 80 \\
\hline Máximo & 32 & 40 & 40 & 40 & 40 & 40 & 40 & 40 \\
\hline P. M. teórico & -.02 & -.17 & -.18 & -.36 & -1.04 & -.12 & -.44 & -.73 \\
\hline Asimetría & -.17 & -.04 & .83 & -.79 & -.38 & .43 \\
\hline Curtosis & .41 & -.09 & -.49 & -.82 & .83 \\
\hline
\end{tabular}

Nota. $\mathrm{AU}=$ autodeterminación; $\mathrm{BE}=$ bienestar emocional; $\mathrm{BF}=$ bienestar físico; $\mathrm{BM}=$ bienestar material; $\mathrm{D}=$ derechos; $\mathrm{DP}=$ desarrollo personal; $\mathrm{IS}$ = inclusión social; $\mathrm{RI}$ = relaciones interpersonales.

En lo relativo a la distribución de los ítems que forman la escala KidsLife, en el análisis de las respuestas dadas por los participantes encontramos que las respuestas más positivas se corresponden con los ítems "Sufre situaciones de violencia, abusos, bullying, acoso, maltrato o negligencia" (D) con una frecuencia de respuesta superior al $98 \%$, "Recibe atención inmediata cuando se encuentra mal" (BF) y "Cuenta con una persona que vela por sus derechos" (D), en los que más del $89 \%$ responde "siempre o casi siempre". Existen otros ítems que presentan altos porcentajes de respuestas positivas, superiores al 75\%, como "Recibe elogios y cumplidos cuando hace algo bien" (BE), "Recibe los apoyos de profesionales sanitarios que necesita, p. e., pediatra, logopeda, fisioterapeuta" (BF), "Tiene en regla toda su documentación personal, prestaciones

(C) Ediciones Universidad de Salamanca / CC BY-NC-ND

Siglo Cero, vol. 47 (3), n. ${ }^{\circ}$ 259, 2016, julio-septiembre, pp. 7-27 
y valoraciones que le corresponden por ley" (D), "El centro respeta la privacidad de la información p. e., difusión de imágenes, datos personales" (D), "En su entorno es tratado/a con respeto" (D) y "Se protege de forma adecuada la confidencialidad de sus evaluaciones individuales" (D).

En el otro extremo, las respuestas más negativas se obtienen en los ítems "Participa en grupos naturales de su comunidad p. e., scouts, deportes, sociales, educativos, religiosos" (IS), "Decora la habitación a su gusto p. e., elige pósters, cuadros, disposición de los objetos" (AU) y "Participa en la elaboración de su plan individual de apoyos" (AU) ya que el porcentaje de respuesta "nunca o casi nunca" supera el $50 \%$. Existen otros ítems que también alcanzan importantes porcentajes de respuesta negativa, superiores al 35\%, como "Elige la ropa que se quiere poner" (AU), "Recibe los apoyos adecuados para gestionar su dinero" (BM), "Se le informa adecuadamente acerca de decisiones importantes que se toman en su nombre" (D) y "Dispone de recursos que facilitan el contacto con sus seres queridos (p. e., teléfono móvil, ayudas técnicas e Internet)" (RI).

En términos generales, el género no dio lugar a diferencias significativas en la puntuación total, aunque las puntuaciones fueron ligeramente superiores en los hombres $(M=502.41 ; D T=52.22)$ que en las mujeres $(M=485.55 ; D T=51.30)$. Tampoco aparecieron diferencias en las puntuaciones por dimensiones, con la única excepción de $\mathrm{BF}\left(t_{(101)}=-2.26 ; p=.02\right)$, en la que los hombres $(M=70.05 ; D T=6.49)$ obtuvieron mejores puntuaciones que las mujeres $(M=67.13 ; D T=6.58)$. Dentro de esta dimensión de $\mathrm{BF}$, los ítems concretos en los que existían diferencias entre hombres y mujeres fueron los relativos a tener una adecuada higiene e imagen personal, recibir atención inmediata cuando se encuentran mal, prestar atención al diagnóstico y tratamiento de las discapacidades sensoriales y saber utilizar las ayudas técnicas necesarias para su movilidad por parte de las personas que les prestan apoyo.

Resultados similares fueron encontrados con la variable edad. El análisis de varianza $(A N O V A)$ no arrojó diferencias significativas en la puntuación total de la escala $\left(F_{(2)}=.18 ; p=.83\right)$, ni en las puntuaciones obtenidas en las diferentes dimensiones.

En cambio, el análisis de la puntuación total en la escala mostró diferencias significativas según el nivel de DI. Si atendemos a las medias observamos que los participantes con DI leve obtienen las puntuaciones más altas $(M=524.14 ; D T=33.20)$, seguidos por el grupo de DI moderada $(M=516.730 ; D T=37.987)$ y severa $(M=491.23$; $D T=50.30$ ), mientras que el grupo de participantes con DI profunda obtienen las puntuaciones más bajas $(M=455.89 ; D T=60.70)$. Los resultados del $A N O V A$ ponen de manifiesto estas diferencias en la puntuación total de la escala en función del nivel de $D I\left(F_{(3)}=6.93 ; p=.00 ; \eta^{2}=.17\right)$, y los resultados de las pruebas post hoc muestran diferencias significativas entre los grupos de DI leve y profunda $(p=.02)$, así como entre DI moderada y profunda $(p=.00)$.

También se obtienen diferencias estadísticamente significativas en seis de las ocho dimensiones: IS $\left(F_{(3)}=2.80 ; p=.04 ; \eta^{2}=.07\right)$, con un tamaño del efecto pequeño; AU $\left(F_{(3)}=4.06 ; p=.00 ; \eta^{2}=.11\right)$ y BM $\left(F_{(3)}=3.93 ; p=.01 ; \eta^{2}=.10\right)$, con tamaños de los efectos medios; y $\mathrm{D}\left(F_{(3)}=8.26 ; p=.00 ; \eta^{2}=.20\right), \mathrm{DP}\left(F_{(3)}=5.19 ; p=.00 ; \eta^{2}=.13\right)$ y RI $\left(F_{(3)}=10.32 ; p=.00 ; \eta^{2}=.23\right)$, con tamaños de los efectos más grandes. Al realizar los

(C) Ediciones Universidad de Salamanca / CC BY-NC-ND

Siglo Cero, vol. 47 (3), n. ${ }^{\circ}$ 259, 2016, julio-septiembre, pp. 7-27 
análisis post hoc para determinar entre qué niveles se dan esas diferencias, sólo aparecen en AU entre DI moderada y profunda ( $p=.01)$; en BM entre DI moderada y profunda ( $p=.01)$; en D entre DI leve y profunda $(p=.01)$, así como entre DI moderada y severa $(p=.03)$ y entre DI moderada y profunda $(p=.00)$; en DP existen diferencias entre DI leve y profunda $(p=.01)$ y entre DI moderada y profunda $(p=.02)$; por último, en RI hay diferencias entre DI leve y profunda $(p=.00)$, DI moderada y severa $(p=.02)$ y DI moderada y profunda $(p=.00)$.

Respecto a la puntuación total en la escala en función del tipo de escolarización, si atendemos a las medias, observamos que son los participantes de educación ordinaria los que obtienen las puntuaciones más altas $(M=521.42 ; D T=33.77)$, seguidos por los participantes de educación combinada $(M=504 ; D T=38.96)$ y educación especial $(M=490.13 ; D T=54.41)$. En términos generales, esta variable no dio lugar a diferencias significativas en la puntuación total $\left(F_{(2)}=2.04 ; p=.13\right)$ ni en las puntuaciones por dimensiones, con la única excepción de IS $\left(F_{(2)}=4.15 ; p=.01 ; \eta^{2}=.07\right)$ en la que el grupo de educación ordinaria obtiene puntuaciones significativamente más altas $(M=47.08 ; D T=6.62 ; p=.02)$ que el grupo de educación especial $(M=40.56 ; D T$ $=7.83$ ). Dentro de esta dimensión, los ítems concretos en los que existían diferencias entre ambos grupos fueron los relativos a realizar actividades de ocio con personas de su edad; participar en actividades inclusivas adecuadas para sus condiciones físicas y mentales; y participar en actividades de ocio en entornos comunitarios y grupos de su comunidad (p. e., scouts, sociales, deportivos, religiosos).

El análisis de la puntuación total de la escala y de las distintas dimensiones según las variables predictoras tipo de enfermedad y comunidad autónoma de residencia no dio lugar a diferencias estadísticamente significativas.

Por último, el análisis de la puntuación total en relación al nivel de necesidades de apoyo reveló la existencia de diferencias estadísticamente significativas $\left(F_{(3)}=6.63 ; p\right.$ $=.00 ; \eta^{2}=.16$ ), ya que el $16.7 \%$ de las diferencias se deben al nivel de necesidades de apoyo. La prueba T3 de Dunnet mostró que esas diferencias tienen lugar entre el grupo de necesidades de apoyo generalizado y los grupos de apoyo limitado $(p=.00)$, intermitente $(p=.00)$ y extenso $(p=.00)$. Del mismo modo, el $A N O V A$ para cada una de las ocho dimensiones que conforman la escala dio lugar a diferencias significativas en AU $\left(F_{(3)}=3.73 ; p=.01 ; \eta^{2}=.10\right) ; \mathrm{BM}\left(F_{(3)}=5.01 ; p=.00 ; \eta^{2}=.13\right) ; \mathrm{D}\left(F_{(3)}=7.41 ; p=.00 ; \eta^{2}=\right.$ $.18) ; \mathrm{DP}\left(F_{(3)}=4.65 ; p=.00 ; \eta^{2}=.12\right)$, y $\mathrm{RI}\left(F_{(3)}=9.43 ; p=.00 ; \eta^{2}=.22\right)$ con tamaños de los efectos medios y grandes. Los análisis post hoc revelaron diferencias significativas ( $p$ $<.05$ ) entre los niveles de apoyo limitado y generalizado en BM, DP y RI. Asimismo, se observaron diferencias $(p<.05)$ entre los niveles de necesidades de apoyo generalizado y extenso en BM, D y RI. Por último, se encontraron diferencias significativas $(p<.05)$ entre los niveles de apoyo generalizado e intermitente en D, DP y RI.

\section{Discusión}

El objetivo principal de este trabajo ha sido estudiar la CV en niños y jóvenes con ER y DI a través de la escala KidsLife, ámbito de conocimiento que apenas ha tenido 
desarrollo investigador. También ha interesado analizar la incidencia de determinadas variables predictoras (género, edad, nivel de DI, tipo de escolarización, tipo de enfermedad y comunidad autónoma) en la variable criterio con el fin último de proporcionar una guía a las organizaciones proveedoras de servicios para la mejora de los apoyos que proporcionan. En líneas generales, los resultados encontrados muestran, por un lado, que la puntuación media de la escala supera ampliamente el punto medio teórico de la misma y que existen diferencias en la influencia que ejercen las variables predictoras estudiadas en la CV de estos niños y jóvenes.

Así, el hecho de que las puntuaciones superen el punto medio teórico de la escala puede parecer, en principio, un resultado un tanto sorprendente. Ello es debido a que en la bibliografía especializada prevalecen los resultados que informan en sentido contrario, es decir, que la CV de estos niños es significativamente más baja que la de sus pares sin ER y DI (Johansen et al., 2013) y que esta se encuentra muy comprometida debido a la importante pérdida de autonomía (Puente-Ferreras et al., 2011). Sin embargo, los resultados que hemos obtenido concuerdan con los obtenidos por otros autores (Caputo, 2014) que aluden a la "paradoja de la discapacidad" para hacer referencia a que las personas que tienen discapacidades persistentes pueden informar de una buena CV debido a que es un constructo muy amplio en el que cada uno establece un balance entre cuerpo, mente y contexto. Por ello, y entre las líneas futuras de investigación, se recomienda seguir indagando las posibles causas de este resultado.

La dimensión IS es la que ha obtenido puntuaciones más bajas, lo que podría estar relacionado con una limitación para participar en algunas actividades o para relacionarse con otras personas debido a la idiosincrasia de la ER y del nivel de DI (Bendixen et al., 2012; CERMI, 2010; Gaite et al., 2008; Johansen et al., 2013; Unicef, 2013a, b). Por el contrario, la dimensión $\mathrm{BF}$ es la que ha obtenido mayores puntuaciones, posiblemente porque durante los últimos años se han producido cambios significativos en la atención y cuidado de estas personas, y el objetivo médico y rehabilitador es más integral y abarca todos los problemas de salud que puedan afectar al niño y producir otras limitaciones asociadas (Gaite et al., 2008). En otras palabras, viene imperando una perspectiva rehabilitadora muy centrada en la reducción de los efectos que provocan las deficiencias en las estructuras y funciones corporales.

Respecto a los ítems, los participantes obtuvieron las puntuaciones más elevadas en el ítem "recibe atención inmediata cuando se encuentra mal" y, por lo tanto, estaría muy relacionado con esa perspectiva rehabilitadora muy centrada en la reducción de los efectos que provocan las deficiencias para así lograr mayor bienestar físico. En la misma línea, el ítem "tiene en regla toda su documentación personal, prestaciones y valoraciones que le corresponden por ley" obtiene puntuaciones superiores respecto a otros ítems, cuestión que no resulta extraña dada la importancia que este aspecto tiene, tanto para los profesionales como para las familias, para el acceso y consecución de ayudas y prestaciones que mejoren la CV de estos chicos. También el ítem "el centro respeta la privacidad de la información" alcanza puntuaciones elevadas, e informa de la importancia que los centros y organizaciones otorgan a la protección y privacidad de los datos. 
Sin embargo, las puntuaciones más bajas se obtuvieron en los ítems "participa en grupos naturales de su comunidad", posiblemente debido a las limitaciones propias de la ER y la DI; "decora la habitación a su gusto"; "participa en la elaboración de su plan individual de apoyos”, lo que podría interpretarse como que son otras personas las que toman las decisiones en su lugar, y "sufre situaciones de violencia, abusos, bullying, acoso, maltrato o negligencia”, que podría relacionarse con la visibilidad y respeto a la integridad que ha conseguido el colectivo durante los últimos años. Todos estos datos se encuentran en la línea de lo apuntado por distintos autores (Arellano y Peralta, 2013; CERMI, 2010; Unicef, 2013a, b), que sostienen que los niños y jóvenes con DI y ER son más vulnerables a la discriminación, exclusión y abuso, del mismo modo que también encuentran más dificultades en la participación social y autonomía personal que sus iguales sin discapacidad.

En cuanto al género, no se han encontrado diferencias en la puntuación total de la escala, en cambio, sí aparecieron diferencias en BF. Parece que los hombres informan de un mayor nivel de bienestar físico que las mujeres, lo que tal vez podría explicarse por el efecto social de la variable género ya que, como señala CERMI (2006, 2010), las mujeres con discapacidad no sólo están entre las más importantes consumidoras de atención médica y sanitaria, sino que también perciben además mayor discriminación en la atención sanitaria que los hombres. En este sentido, Unicef (2013b) informa que el género es un factor crucial de exclusión ya que las niñas y jóvenes con discapacidad sufren una doble discriminación, en primer lugar, por la propia discapacidad y, en segundo lugar, por el hecho de ser mujer, lo que dificulta el acceso a los servicios de salud y repercute en su bienestar físico. Sin embargo, estos resultados no concuerdan con los obtenidos en otros estudios en los que no se encontraron diferencias en función del género (Sprangers et al., 2000).

Respecto a la $e d a d$, no se encontraron diferencias estadísticamente significativas en la puntuación total de la escala ni en las diferentes dimensiones que la conforman. Estos resultados concuerdan con los obtenidos por Bendixen et al. (2012), aunque otros autores (Sprangers et al., 2000) informan de puntuaciones más bajas en CV en los participantes de mayor edad respecto a los más jóvenes en la dimensión BF. Esto podría deberse a que en este trabajo la diferencia en el rango de edad de los participantes no era tan elevada como en el de Sprangers et al., por tanto, cabría esperar que la gravedad de las condiciones clínicas asociadas de los participantes en este estudio fuera menor, implicando por tanto una mayor CV. Del mismo modo, el informe del CERMI (2010) recoge que es a partir de los veinte o treinta años cuando la población con discapacidad encuentra más dificultades para acceder a recursos que facilitan una vida autónoma.

Por otro lado, según el nivel de DI se encontraron diferencias significativas en la puntuación total de la escala, así como en seis de las ocho dimensiones (AU, BM, D, DP y RI). Estas diferencias aparecen principalmente entre los niveles de DI leve y profunda y también entre DI moderada y profunda. Estos resultados coinciden con los obtenidos por CERMI (2010) y Unicef (2013a, b) que plantean que a mayor nivel de DI mayor dificultad para participar en actividades sociales y recreativas, mayor sobreprotección y menor autonomía personal. 
El análisis en función del tipo de escolarización no arroja diferencias significativas respecto a la puntuación total de la escala, pero sí aparecen estas diferencias en la dimensión IS, ya que los participantes de educación ordinaria obtienen puntuaciones más altas que aquellos que están integrados en educación especial. Esto permite afirmar que la educación inclusiva favorece una mayor CV pues posibilita que los niños con discapacidad compartan la misma experiencia educativa que sus iguales. En este sentido, Jenaro, Vega, Flores y Cruz (2013) señalan la importancia de proporcionar más oportunidades para que las personas con DI sean incluidas en los servicios y organizaciones entre los que se encuentra la escuela, ya que hay una estrecha relación entre inclusión, satisfacción personal y CV. En esta línea, tal y como sostiene Unicef (2013a), sería necesario que los centros ordinarios garanticen los apoyos y ajustes necesarios para atender adecuadamente a las diversas necesidades educativas que tienen los niños y jóvenes con discapacidad, lo que facilitaría que la inclusión educativa sea verdaderamente efectiva.

En cuanto al tipo de enfermedad, no se encontraron diferencias estadísticamente significativas en la puntuación total de la escala ni en las ocho dimensiones de la misma. Este resultado podría ser explicado, tal y como sostienen algunos autores (Bendixen et al., 2012; Caputo, 2014), porque el constructo CV no sólo implica aspectos objetivos, sino también subjetivos, de forma que cada uno realiza un balance de su propia vida. En este sentido, debemos tener en cuenta que, dado que la significación depende en gran medida del tamaño de la muestra, no podemos afirmar la inexistencia de diferencias en muestras más amplias.

Otra de las variables predictoras estudiadas, la comunidad autónoma de residencia, no estableció diferencias significativas en la puntuación total ni en las ocho dimensiones de la escala. Puente-Ferreras et al. (2011) refieren que hay ER que son frecuentes en una parte del mundo, pero infrecuentes en otras y, en la misma línea, Karr (2011) sostiene que el lugar de residencia no es un buen predictor de la CV.

Por último, el análisis según el nivel de necesidades de apoyo evidencia diferencias significativas tanto en la puntuación total de la escala como en cinco de las dimensiones (AU, BM, D, DP y RI). Por tanto, parece que la CV se relaciona con el nivel de necesidades de apoyo, ya que son aquellos que necesitan menos apoyo los que obtienen mayores puntuaciones y, a su vez, refieren mayor bienestar, desarrollo personal, autonomía, autodeterminación personal, inclusión y participación social. Resultados similares son recogidos por Puente-Ferreras et al. (2011), al afirmar que las ER con frecuencia obligan tanto a la persona que la padece como a su entorno a modificar su vida, ya que requieren cuidados y apoyos especializados y muy prolongados, lo que repercute en su autonomía, bienestar, desarrollo y, por tanto, en su CV.

En definitiva, este trabajo presenta como uno de sus puntos fuertes el haberse centrado en la evaluación de la $\mathrm{CV}$ de uno de los colectivos de discapacidad más olvidados en la investigación: los niños y jóvenes con ER y DI. Constituye, por tanto, un primer paso para conocer la problemática y necesidades de esta población de forma que permita la implementación de prácticas basadas en la evidencia con el fin de mejorar su CV. Asimismo, los resultados encontrados aportan importantes evidencias de fiabilidad que avalan la consistencia interna de la escala KidsLife, instrumento pionero en 
nuestro país en la evaluación de la CV en personas con DI que están en sus primeras fases del ciclo vital.

No obstante, como toda investigación, este trabajo adolece también de varios puntos débiles. En cuanto a la muestra, y dado que no pudo ser seleccionada al azar, se encuentra sesgada debido a su carácter de conveniencia, de ahí que las conclusiones no puedan ser generalizables a la población de personas con ER y DI. Por otro lado, el tamaño muestral no es demasiado grande, aunque conviene señalar que dadas las características específicas de la misma no resulta sencillo disponer de muestras más amplias. Cabría señalar, como otra de las limitaciones de este trabajo, el hecho de que la evaluación de la CV de los participantes haya sido realizada por otros informantes, dadas las limitaciones que estas personas tienen para aportar información sobre sí mismas (Gómez et al., 2014). Ello hace que se recurra al heteroinforme como alternativa de evaluación, a pesar de que esta pueda estar sesgada, ya que no siempre existe elevada concordancia entre las percepciones y opiniones de terceras personas y las emitidas por la propia persona con discapacidad (Balboni, Coscarelli, Giuntia y Schalock, 2014; Verdugo et al., 2013).

Para finalizar, los resultados de este trabajo aportan información que puede resultar útil para el diseño e implementación de prácticas que permitan mejorar los resultados personales de los niños y jóvenes con DI y ER en lo relativo a su autodeterminación, inclusión y relaciones interpersonales, principalmente. Son personas más vulnerables a sufrir situaciones de abuso y discriminación, con una limitada autonomía y mayores dificultades para la participación e inclusión social que sus pares sin discapacidad. Es necesario continuar investigando en esta temática de forma que pueda lograrse un amplio cuerpo de conocimientos sobre los que planificar programas de prestación de servicios eficaces, desarrollar estrategias para disminuir su discriminación, potenciar las oportunidades para una educación inclusiva y favorecer, en definitiva, su pleno desarrollo como personas autónomas. En esta línea, tal y como apunta Unicef (2013a, b), algunas actuaciones recomendadas serían realizar estudios sobre los problemas concretos que afectan a los jóvenes con discapacidad, adoptar medidas políticas y legales para disminuir la vulnerabilidad del colectivo, eliminar los centros de educación especial y convertirlos en centros de recursos de apoyo para que los niños y niñas con discapacidad puedan tener una educación inclusiva en centros ordinarios, proporcionar apoyos para que puedan participar en la toma de decisiones que les afecten, evitar la institucionalización y promover políticas de apoyo a las familias de jóvenes con discapacidad.

\section{Agradecimientos}

Este estudio ha sido posible gracias a la financiación del Ministerio de Economía y Competitividad (PSI2012-33139). El proyecto recibió la aprobación del Comité de Ética de la Universidad de Oviedo. Los autores agradecen sinceramente la participación de las personas con discapacidad intelectual, sus familias y los profesionales que les proporcionan apoyos en organizaciones y centros escolares, así como a Plena Inclusión por su apoyo en este proyecto. 


\section{Referencias bibliográficas}

Alcedo, M. A., Aguado, A. L., Arias, B., González, M. y Rozada, C. (2008). Escala de Calidad de Vida (ECV) para personas con discapacidad que envejecen: estudio preliminar. Intervención Psicosocial, 17 (2), 153-167.

Arellano, A. y Peralta, F. (2013). Calidad de vida y autodeterminación en personas con discapacidad. Valoraciones de los padres. Revista Iberoamericana de Educación, 63, 145-160.

Balboni, G., Coscarelli, A., Giuntia, G. y Schalock, R. L. (2014). The assessment of the quality of life of adults with intellectual disability: The use of self-report and report of others assessment strategies. Research in Developmental Disabilities, 34, 4248-4254.

Bendixen, R. M., Senesac, C., Lott, D. J. y Vanderborne, K. (2012). Participation and quality of life in children with Duchenne muscular dystrophy using the International Classification of Functioning, Disability, and Health. Health and Quality of Life Outcomes, 10, 43-51.

Caliandro, P., Grugni, G., Padua, L., Kodra, Y., Tonali, P., Gargantini, L., ...y Taruscio, D. (2007). Quality of life assessment in a simple of patients affected by Prader-Willi syndrome. Journal of Pediatrics and Child Health, 43, 826-830.

CAputo, A. (2014). Exploring quality of life in Italian patients with rare disease: a computeraided content analysis of illness stories. Psychology, Health and Medicine, 19 (2), 211-221.

Cermi (2006). Discapacidad y asistencia sanitaria. Recuperado de http://www.feaps.org/biblioteca/documentos/asistencia_sanitaria.pdf.

Cermi (2010). Los jóvenes con discapacidad en España. Informe de situación. Madrid: Cinca, S. A. Recuperado de http://www.sindromedown.net/adjuntos/cEnlacesDescargas/ 438_1_los.pdf.

Cummins, R. A. (2000). Objective and subjective quality of life: an interactive model. Social Indicators Research, 52, 55-72.

European Union CommitTee of Experts on Rare Diseases (2014). Report on the state of the art of rare disease activities in Europe. Recuperado de http://www.eucerd.eu/upload/file/ Reports/2014ReportStateofArtRDActivities.pdf.

FEDERACión EsPañola de EnFERMEDAdes RARAs (2015). ¿Qué son las enfermedades raras? Recuperado de http://www.enfermedadesraras.org/index.php/enfermedades-raras/enfermedades-raras-en-cifras.

Felce, D. y Perry, J. (1995). Quality of life: Its definition and measurement. Research in Developmental Disabilities, 16 (1), 51-74.

Gaite, L., García Fuentes, M., González Lamuño, D. y Álvarez, J. L. (2008). Necesidades en las enfermedades raras durante la edad pediátrica. Anales del Sistema Sanitario de $\mathrm{Na}$ varra, 31, 165-175.

Gómez, L. E. (2010). Evaluación de la calidad de vida en servicios sociales: Validación y calibración de la Escala Gencat. Tesis doctoral. Universidad de Salamanca, Instituto Universitario de Integración en la Comunidad (INICO).

Gómez, L. E., Alcedo, M. A., Arias, B., Fontanil, Y., Arias, V. B., Monsalve, M. A. y Verdugo, M. Á. (2016). A new scale for the measurement of quality of life in children with intellectual disability. Research in Intellectual and Developmental Disabilities, 5354, 399-410.

Gómez, L. E., Alcedo, M. A., Monsalve, A. y Fontanil, Y. (2014). The KidsLife Scale: a tool for guiding quality of life improvement in human services. Póster en 138 AAIDD Annual Meeting and Conference, Embracing complexity, Inclusion, Participation, and Citizenship. Orlando, Florida, Estados Unidos. 
Gómez, L. E., Alcedo, M. A., Verdugo, M. Á., Arias, B., Arias, V., Monsalve, A., Fontanil, Y. y Morán, L. (2016). KidsLife: evaluación de la calidad de vida de niños para niños y adultos jóvenes. Salamanca: INICO.

Gómez, L. E., Arias, V., Morán, L., Alcedo, M. A., Arias, B. y Verdugo, M. Á. (2015). Quality of life of children outcomes of children with intellectual disability. Póster en 139 AAIDD Annual Meeting and Conference, Making a Difference Through Research, Practice, and Policy. Louisville, Kentucky, Estados Unidos.

Gómez, L. E., Peña, E., Alcedo, M. A., Monsalve, A., Fontanil, Y., Verdugo, M. Á., Arias, V. B. y Arias, B. (2014). El constructo de calidad de vida en niños y adolescentes con discapacidades múltiples y profundas: propuesta para su evaluación. Siglo Cero, 45 (1), 56-69.

Gómez, L. E., Peña, E., Arias, B. y Verdugo, M. Á. (2016). Impact of Individual and Organizational Variables on Quality of Life. Social Indicators Research, 125 (2), 649-664.

Gómez, L. E., Verdugo, M. Á. y Arias, B. (2010b). Calidad de vida individual: Avances en su conceptualización y retos emergentes en el ámbito de la discapacidad. Behavioral Psycho$\log y, 18(3), 453-472$.

Gómez, L. E., Verdugo, M. Á., Arias, B. y Navas, P. (2010a). Consideraciones conceptuales y metodológicas sobre el desarrollo de escalas multidimensionales de calidad de vida centradas en el contexto. Siglo Cero, 41 (2), 59-80.

Gómez, L. E., Verdugo, M. Á., Arias, B. y Arias, V. (2011). A comparison of alternative models of individual quality of life. Social Indicators Research, 101, 109-126.

Gómez-Vela, M. y Verdugo, M. Á. (2009). Cuestionario de evaluación de la calidad de vida de alumnos adolescentes: manual de aplicación. Madrid: CEPE.

Instituto Nacional de Estadística (2008). Encuesta de Discapacidad, Autonomía personal y situaciones de Dependencia (EDAD). Recuperado de http://sid.usal.es/estadisticas_edad2008.asp.

Jenaro, C., Vega, V., Flores, N. y Cruz, M. (2013). Quality of services and quality of life from service providers' perspectives: analysis with focus groups. Journal of Intellectual Disability Research, 57, 489-499.

Jenaro, C., Verdugo, M. Á., Caballo, C., Balboni, G., Lachapelle, Y., Otbrebski, W. y SCHALOCK, R. L. (2005). Cross-cultural study of person-centered quality of life domains and indicators: a replication. Journal of Intellectual Disability Research, 49, 734-739.

Johansen, H., Dammann, B., Andresen, I. L. y Fagerland, M. (2013). Health-related quality of life for children with rare diagnoses, their parents satisfaction with life and the association between the two. Health and Quality of Life Outcomes, 11, 152-159.

KARR, V. L. (2011). A life of quality: Informing the UN Convention on the rights of persons with disabilities. Journal of Disability Policy Studies, 22 (2), 67-82.

López, J., Linertová, R., Serrano, P., Hens, M., Posada, M. y Oliva, J. (2012). Los costes socioeconómicos y la calidad de vida relacionada con la salud en pacientes con enfermedades raras en España. Proyecto de IMSERSO n..$^{\circ}$ 167/10. Ministerio de Sanidad, Política Social e Igualdad. Recuperado de http://www.imserso.es/InterPresent1/groups/imserso/documents/binario/010_167idi.pdf.

Mura, G., Krishna, M. B., Pisano, A., Licci, G. y Carta, M. G. (2012). Psychiatric symptoms and quality of life in systemic sclerosis. Clinical Practice and Epidemiology in Mental Health, 8, 30-35.

Petry, K., Maes, B. y Vlaskamp, C. (2005). Domains of quality of life of people with profound multiple disabilities: The perspective of parents and direct support staff. Journal of Applied Research in Intellectual Disabilities, 18, 35-46. 
Picci, R. L., Oliva, F., Trivelli, F., Carezana, C., Zuffranieri, M., Ostacoli, L., ... y Lala, R. (2015). Emotional burden and coping strategies of parents of children with rare diseases. Journal of Children and Family Studies, 24, 514-522.

Puente-Ferreras, A., Barahona-Gomariz, M. J. y Fernández-Lozano, M. P. (2011). Las enfermedades raras: naturaleza, características e intervención biopsicosocial. Portularia, 9 (1), 11-23.

Sabeh, E., Verdugo, M. Á., Prieto, G. y Contini, N. (2009). CVI-CVIP. Cuestionario de evaluación de la calidad de vida en la infancia. Madrid: CEPE.

SCHAlOCK, R. L. y VeRdugo, M. Á. (2002). Quality of life for human service practitioners. Washington, DC: American Association on Mental Retardation [Traducido al castellano por M. Á. Verdugo y C. Jenaro (2003). Calidad de vida. Manual para profesionales de la educación, salud y servicios sociales. Madrid: Alianza].

Schalock, R. L. y Verdugo, M. Á. (2007). El concepto de calidad de vida en los servicios y apoyos para personas con discapacidad intelectual. Siglo Cero, 38, 21-36.

SCHAlock, R. L., Verdugo, M. Á. y GÓmez, L. E. (2011). Evidence-based practices in the field of intellectual and developmental disabilities: An international consensus approach. Evaluation and Program Planning, 34, 273-282.

Schalock, R. L., Verdugo, M. Á., Jenaro, C., Wang, M., Wehmeyer, M., Xu, J. y LachaPELle, Y. (2005). A cross-cultural study of quality of life indicators. American Journal on Mental Retardation, 110, 298-311.

Sprangers, M., Regt, E., Andries, F., Van Agt, H., Bijl, R., De Boer, J., ... y De Haes, H. (2000). Which chronic conditions are associated with better or poorer quality of life? Journal of Clinical Epidemiology, 53, 895-907.

UNICEF (2013a). Estudio sobre la situación de niños y niñas con discapacidad en España. Barcelona: Huygens. Recuperado de http://www.unicef.es/sites/www.unicef.es/files/libro_02_ web.pdf.

Unicef (2013b). Estado Mundial de la Infancia. Niños y niñas con discapacidad. Recuperado de http://www.unicef.org/lac/SOWC2013_fullreport_esp(8).pdf.

Verdugo, M. Á., Gómez, L. E., Arias, B., Navas, P. y Schalock, R. L. (2014). Measuring quality of life in people with intellectual and multiple disabilities: Validation of the San Martín Scale. Research in Developmental Disabilities, 35, 75-86.

Verdugo, M. Á., Gómez, L. E., Arias, B., Santamaría, M., Clavero, D. y Tamarit, J. (2013). Evaluación de la calidad de vida en personas con discapacidades intelectuales o del desarrollo: la escala INICO-FEAPS. Siglo Cero, 44 (3), 6-20.

Wang, M., Schalock, R. L., Verdugo, M. Á. y Jenaro, C. (2010). Examining the factor structure and hierarchical nature of the quality of life construct. American Journal on Intellectual and Developmental Disabilities, 155 (3), 218-233. 


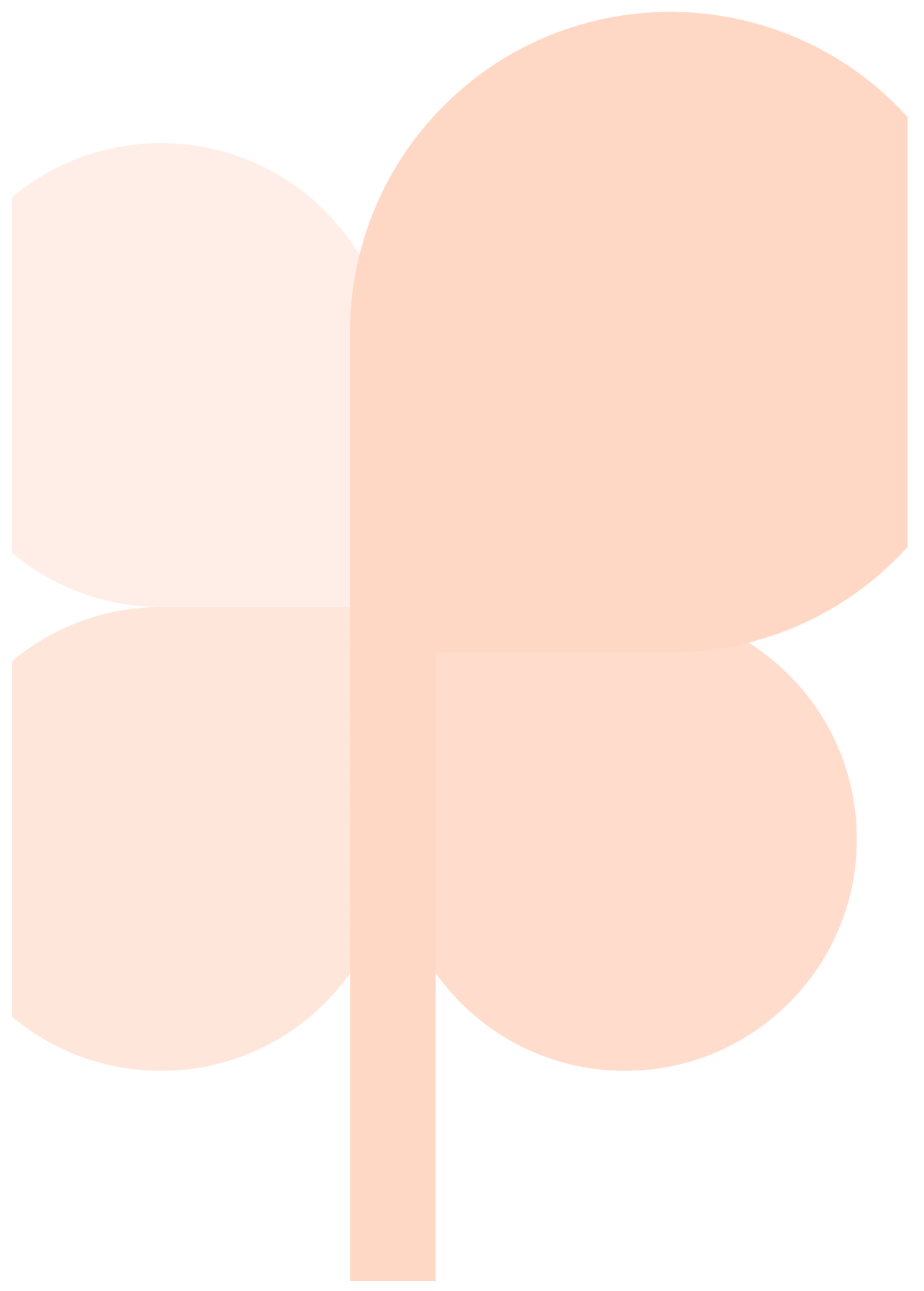

